\title{
XII.
}

\section{Orpheus in der Unterwelt.}

\author{
Orpheum nescio quomodo infernis sacris \\ praeficere soleat civitas impiorum. \\ Augustinus.
}

Die von mir im Jahrbuch des Deut. archäol. Instituts VIII (1893) S. $104 \mathrm{ff}$. vorgeschlagene Deutung der unteritalischen Unterweltsdarstellungen hat Milchhöfer in dieser Zeitschrift LIII S. $385 \mathrm{ff}$. angegriffen und ihre Unhaltbarkeit nachweisen zu können geglaubt. Es ließ sich voraussehn, daß eine Deutung, die ein in der Archäologie seit Jahrzehnten geltendes Dogma zu beseitigen suchte, nicht ohne Widerspruch bleiben würde; um so mehr fühle ich mich veranlaßt, sie gegen Angriffe zu schützen, die ihren Ausgangspunkt im Grunde wieder von jener Anschauung aus nehmen, der man heutzutage nicht mehr als eine historische Berechtigung zuerkennen darf.

Milchhöfer setzt in seiner Polemik (S. 386) ein bei der Stellung, die ich Orpheus in diesen Vasengemälden angewiesen habe. Ich faßte ihn als den Stifter der nach ihm benannten Mysterien, wie er für die durch seine Weihen geläuterten bei Persephone um ein seliges Leben bittet. Diese Stellung "als Mittler für die armen Seelen" vergleicht er der eines katholischen Heiligen, fragt, ob ein solches Verhältnis in griechischer Anschauung überbaupt möglich sei und verneint das mit Bestimmtheit. Als Sagenheros sei Orpheus aus dem bekannten Philologus LIV (N. F. VIII), 2. 
Motiv - nämlich um Eurydike zu erbitten - in die Unterwelt gestiegen; "auf Grund seiner Erfahrungen" habe er den Hörern und Gläubigen die verschiedenen Wege der Seligkeit gewiesen; kraft „ihrer" Weihen träten dann die "orot im Hades mehr oder minder zuversichtlich auf, ohne eine Zwischeninstanz, die auch in den Goldtäfelchen nicht erwähnt werde. Dem Hadesgang des Orpheus könne also auch eine andre als die mythische Bedeutung nicht beigelegt sein, er könne sich nur auf Eurydike beziehn, deren Darstellung daher auf den Vasengemälden für "den Kenner des Mythos" durchaus nicht erforderlich gewesen sei.

Diese Entgegnung ist in Rücksicht auf die litterarische wie die künstlerische Ueberlieferung gleich befremdend. Ist Orpheus für die in seine Mysterien eingeweihten wirklich nicht mehr gewesen als der Sagenheros, der vor Zeiten in die Unterwelt hinabgestiegen war und dessen Erfahrungen auf dieser Reise man sich dereinst beim eignen Gang dorthin zu Nutze machen wollte? Hat er nur beschrieben, was er dort sah und jedem danach zu richten sich überlassen? Sind die Weihen der Orphiker nicht orphische Weihen, verlassen sich die Gläubigen nicht auf deren Kraft und damit in letzter Linie auf Orpheus? Wer ist ihr Bürge dafür, daß die Mysterien und der ganze daran sich

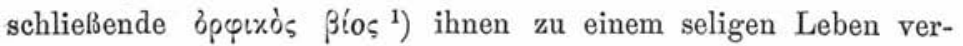
helfen werde, wenn nicht ihr Stifter Orpheus? Was lag den Angehörigen der orphischen Sekte viel an dem Gang ihres Stifters um Eurydike ${ }^{2}$ )? Ihr eigenes Heil lag ihnen am Herzen, deßhalb ließen sie sich einweihen, um selbst auf Grund dieser von Orpheus eingerichteten Weihen ein seliges Leben zu erlangen; das verbürgten ihnen die Weihepriester unter dem Namen

1) Plato Nomoi 6, 782 C. Vgl. Lobeck Agl. I $244 \mathrm{ff}$.

$\left.{ }^{2}\right)$ Ich bemerke übrigens, daß ich von einem $\mathrm{G}$ ang des Orpheus in den Hades gar nicht gesprochen habe und daran bei der Erklärung der Vasenbilder auch gar nicht denken konnte: Orpheus ist im Hades, wie auf dem polygnotischen Gemälde. So wahrscheinlich

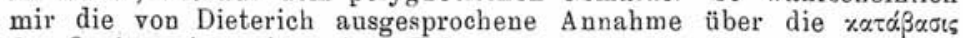
des Orpheus ist, mit meinen Folgerungen hat sie nichts zu thun; ich habe den traditionellen Hadesgang des thrakischen Sängers mit keinem Wort angefochten, überhaupt gar nicht berührt und durfte also nicht erwarten, ein gegen Dieterichs Vermuthung gerichtetes Argument zugleich gegen meine allein an die Vasenbilder sich haltenden Erörterungen von Milchhöfer S. 387 verwerthet zu finden. 


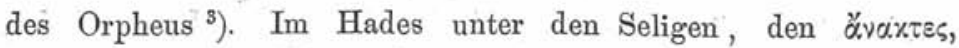
dachten sie ihren Herrn, dorthin ebenfalls als ăvaxtes zu gelangen war das Ziel ihres òpọixòs ßíos.

Sähe man im übrigen nicht das Gegentheil, man müßte wirklich meinen, Milchhöfer hätte sich mit der Litteratur über die Orphiker von Lobeck bis Rohde nur wenig vertraut gemacht; wollte ich diese Dinge weiter ausführen, so könnte ich nicht viel mehr als andere ausschreiben. Ich begnüge mich, eine kurze Stelle Rohdes (Psyche S. 418) hierherzusetzen, eine Zusammenfassung seiner vorangegangenen Ausführungen, die mir deßhalb willkommen ist, weil sie bis auf den Ausdruck wie eine Antwort auf Milchhöfers Frage lautet: „Es bedarf der Offenbarungen und Vermittlungen Orpheus des Gebieters, um den Weg zum Heil zu finden und ängstlicher Beobachtung sei-

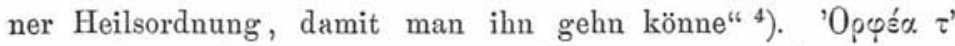

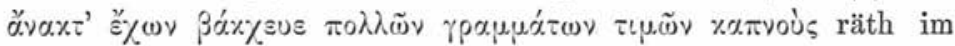
Hippolytos des Euripides (953/4) Theseus seinem Sohn: Orpheus ist der Mittler, der den Weg zum Heil weisen soll, er, der sich auf eine durch Apollo ihm zu Theil gewordene Offenbarung berufen konnte (Lobeck Agl. I 469), der Stifter einer Religion, die nicht nur auf Erfahrungen bei einer Hadesfahrt um Eurydike sich stïtzte. Die orphische Lehre von der Kraft der Fürbitte für Verstorbene (Rohde Psyche S. 421 A. 3) trägt gewiß - von protestantischem Standpunkt aus - einen katholischen Charakter, und doch haben sich Griechen zu ihr bekannt und Milchhöfer hat das nicht zu bestreiten versucht (S. 386 A. 2). Aber wie ist die Macht dieser Fürbitte anders erklärlich als durch eine Vermittlung des Orpheus in der Unterwelt? Orpheum nescio quomodo infernis sacris praeficere soleat civitas impiorum sagt Augustin de civ. dei XVIII 14 (vgl. Lobeck Agl. I 236 A. c.). Dieser Charakter des Orpheus hat zu seiner Parallele mit Christus geführt und deßhalb begegnet uns der Heros später in den christlichen Katakomben, als der Bürge und Ver-

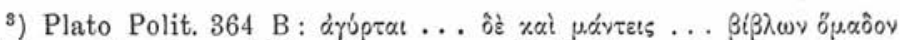

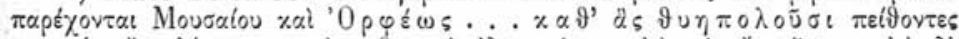

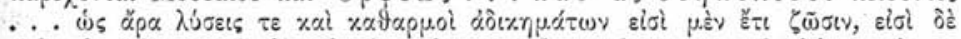

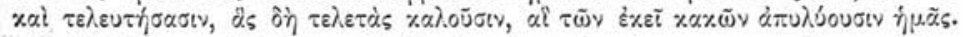

4) Man vergleiche auch die Auseinandersetzungen von Usener über $\pi \alpha \rho \alpha ́ x \lambda \eta \tau o s$ Archiv f. lat. Lexikogr. II (1885) S. 231. 
mittler eines seligen Lebens nach dem Tode ${ }^{5}$ ). Wenn auf den unteritalischen Goldtäfelchen von Orpheus nicht die Rede ist, so möchte ich darauf hinweisen, daß wir bis jetzt gerade vier besitzen und zwar ganz unvollständige; wer weiß zum Beispiel,

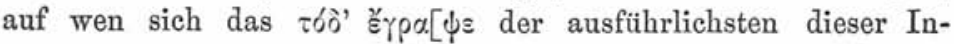
schriften (Kaibel IGSI nr. 641) bezogen haben mag?

$\mathrm{Da}$ ein Grieche oder ein griechisch gebildeter in dem leierspielenden Orpheus der Vasenbilder nur den um Erhörung flehenden Gatten erblickt haben kann (S. 387), ist eine Behauptung, die sich unter diesen Umständen wunderbar ausnimmt, um so wunderbarer, als die sicheren uns erhaltenen Darstellungen der Eurydikesage Orpheus gerade nicht leierspielend, sondern seine Gattin entweder aus dem Hades führend oder von ihr Abschied nehmend zeigen. Wenn aber Milchhöfer nun noch weiter geht und sogar behauptet, für den Kenner des Mythos wäre die Anwesenheit der Eurydike durchaus nicht erforderlich gewesen, so möchte ich ihn darauf hinweisen, daß es keine Eigenthümlichkeit griechischer Kunst ist, im Vertrauen auf die "Kenner" wesentliche Bestandtheile fortzulassen; sie bestrebt sich mit den ihr zu Gebote stehenden Mitteln klar darzustellen, und wenn ein Künstler klar darstellen will, daß Orpheus im Hades um Eurydike bittet, so malt er das Objekt des Wunsches und macht den Sachverhalt nicht etwa durch eine Reihe Eingeweihter noch dunkler. Unzweideutig spricht die Darstellung E III 2: da hält der Sänger seine Eurydike am Arm, kein Eingeweihter ist hier zu finden, weil es sich hier nicht um die Einführung eines Mysten sondern um die Gattin handelt. Wenn Milchhöfer seine Betrachtung mit den Worten schließt, Dieterich und ich hielten diese Darstellung (und eine zweite, auf die ich später zurückkomme), von den übrigen getrennt „weil Eurydike auf den hautsächlich in Betracht kommenden Vasenbildern fehle" (S. 388) und wenn er weiter eine solche Unterscheidung ganz gleichartiger Denkmäler unmethodisch und willkürlich findet, so möchte ich ihn bitten, bei einer etwaigen Fortsetzung seiner Polemik sich nur an mich zu halten ${ }^{6}$ ) und mich

5) Vgl. A. Heußner, die altchristl. Orpheusdarstellungen, Cassel 1893 , S. $24-26,36,37$.

$\left.{ }^{6}\right)$ Ich glaube meine Ansicht auf S. 105 meines Aufsatzes klar genug dargelegt zu haben. 
nicht für Worte Dieterichs verantwortlich zu machen, die dieser weniger scharf gefaßt hat, da er keine Beweisführung mehr erstrebte, sondern sich auf meine Darlegungen berief. Ich glaube, es heißt methodisch verfahren, wenn man Darstellungen des Orpheus mit Eurydike und Darstellungen des Orpheus mit Eingeweihten, die also in dem Punkt auf den es uns ankommt gerade nicht gleichartig sind, trennt. Denn Vasenbilder, auf denen Eurydike und Eingeweihte zusammen erscheinen, sind bisher unbekannt und werden es vermuthlich auch bleiben. Es ist methodisch, wenn ich weiter geschlossen habe: Die einzige Darstellung, auf der mit Sicherheit des Orpheus Aufenthalt im Hades um seiner Gattin willen dargestellt ist, zeigt uns Eurydike, wie das auch nach der Ausdrucksweise der antiken Kunst bestimmt zu erwarten ist; finden wir nun auf anderen Darstellungen Orpheus ohne sie, so werden wir nicht nur die Möglichkeit zu erwägen sondern sogar die Forderung zu stellen haben, da $B$ des Orpheus Anwesenheit hier einen anderen Grund habe und diesen zu ermitteln suchen. Andernfalls hätten wir zu constatieren, daß der Maler sich undeutlich ausgedrückt habe; aber das erfordert einen Beweis, nicht die Ausflucht, daß der Kenner darüber nicht habe im $Z_{\text {weifel }}$ sein können. Gerade bei diesen Gemälden (E I und E III 1) wäre die Darstellung der Eurydike auch für Kenner unumgänglich nöthig gewesen, da sich auf ihnen noch Eingeweihte finden, auf dem einen ein Jüngling, auf dem anderen unmittelbar hinter Orpheus eine Familie; denn daß thatsächlich Eingeweihte in diesen Personen zu erkennen sind, soweit habe ich auch Milchhöfer überzeugt (vgl. S. 390), wenngleich er die Möglichkeit, daß es auch Heroen sein könnten, immer noch nicht für ganz ausgeschlossen hält (S. 389). Nun vergegenwärtige man sich die Sachlage: In Unteritalien sind diese Vasenbilder im vierten Jahrhundert entstanden, in derselben Gegend, in der nach dem Ausweis der Goldtäfelchen die orphische Religion im vierten und dritten Jahrhundert weit verbreitet war. Wer konnte in dieser Gegend auf einem Bild, das Eingeweihte und Orpheus vor den Unterweltsgottheiten zeigte, etwas anderes erkennen, als den Stifter der Mysterien, der für seine Mysten bei Persephone um ein seliges Leben bat? Wer dachte hier an Eurydike, wer konnte an sie denken, die gar nicht einmal dargestellt war? „Es kann sich 
nur um Eingeweihte des eleusinisch-attischen als des populärsten Mysterienkultes handeln, wie sie auch Polygnot verherrlichte", behauptet Milchhöfer S. 390. Man kann sich eines gerechten Staunens ïber die Unteritaliker nicht erwehren, die, obwohl sie zu Eleusis in gar keinen Beziehungen standen, bei ihnen vielmehr die orphischen Mysterien so tief in alle Volksschichten eingedrungen waren, daß wenig über ein Jahrhundert später den Römern ein Senatusconsult dagegen nöthig schien, in ihrem eignen Land und für sich Eingeweihte aus Eleusis im Hades malen, dazu den leierspielenden Orpheus, bei dem sie nur an den Sagenheros denken können, der in den Hades stieg um seiner Gattin willen, die darzustellen endlich nicht für nöthig befunden wurde. So lautet das Ergebnis von Milchhöfers Methode.

Allein selbst zu diesem Ergebnis war noch eine andre Voraussetzung nöthig, die nämlich, daß die Eingeweihten "natïrlich als Insassen des Hades, nicht als Ankömmlinge ${ }^{7}$ ) aufzufassen wären, und Milchhöfer bemüht sich daher, ihre Geberden und ihre Haltung dementsprechend zu erklären. Mit den Gesten hat es auf den unteritalischen Vasenbildern seine eigene Bewandtnis; ich würde diesen allein kein Gewicht beigelegt haben, wie ich mich denn vor einem Schluß aus der Handbewegung der einen Danaide auf E III 1 wohl gehütet habe. Aber es ist eine unzweideutige Charakteristik, wenn der Jüngling auf demselben Gemälde mit einem Stock und mit Schuhen ausgerüstet erscheint: der Künstler bezeichnete damit, daß er ihn auf einer Reise dachte. Der Wanderer steht am Ende derselben, vor dem Palast der Unterweltsgöttin - was kann er da anders thun, als der Persephone seine Bitte vortragen? Ist das zweifellos, so darf man aus dieser Situation heraus auch wohl die Haltung seines Arms erklären. Noch klarer spricht das Gemälde E VI 2, auf dem Orpheus fehlt. Wieder stützt sich der Jüngling auf einen Stock, beide Arme hat er erhoben, ist also ohne Zweifel in einem Gespräch mit Jemand begriffen. Daß

7) Ich constatiere hierbei, daß diese Bezeichnung Milchhöfer angebört, obgleich er sie auf S. 389 in einer dem Leser Freude bereitenden Weise durch das Pronomen possessivum und Anführungszeichen mir vindiciert. Diese Form pflegt man nur wörtlichen Citaten zu geben. 
er sich zu den Unterweltsgottheiten wendet, hat kein unbefangener Erklärer der Vase in Frage gestellt ${ }^{8}$ ), nach der Richtung seines Hauptes schon ist es ganz zweifellos. Milchhöfer darf das nicht zugeben und läßt ihn delshalb mit der oberhalb von ihm sitzenden Frau („doch wohl Aphrodite “) ${ }^{9}$ ) sich" unterhalten. Ich will nicht weiter Gewicht darauf legen, daß er zu ihr gar nicht hinsieht, was nach den beiden Abbildungen im Bull. Nap. N. S. III tav. 3 und in den Wiener Vorlegeblättern ganz deutlich ist; was aber denkt sich Milchhöfer als den Inhalt dieser in sich doch ganz abgerundeten Darstellung? Das hat er anzugeben unterlassen; und doch hat der Maler einen bestimmten Vorgang darstellen wollen, mag er auch noch so viele Typen aus andern Werken herübergenommen haben, auf denen sie ursprünglich eine ganz andere Bedeutung hatten ${ }^{10}$ ).

Leider ist mir - und andern ist es nicht besser ergangen - auch nicht klar geworden, wie Milchhöfer die beiden andern Unterweltsbilder mit Orpheus auffaßt. Orpheus läßt er dem Gedanken nach mit den übrigen Situationsbildern, die zur Ausschmïckung des Hades dienen, vollkommen in derselben Reihe stehn; er erkennt ein Bestreben, möglichst viele Gattungen von Hadesbewohnern vorzuführen (S. 387). Was wollten die apulischen Künstler? Nur viele Hadesbewohner zeigen? Diese Auffassung legt die Sparsamkeit der Maler von E VI 2 und E V nicht grade nahe. Nur Situationsbilder geben? Dann hätten sie Orpheus und Herakles in Ruhe dargestellt, nicht handelnd; ihre Thätigkeit ist eine andre, als die ewig währende des Sisyphos und Tantalos, für die ich mir die Bezeichnung Situationsbilder noch gefallen lasse. Herakles entführt den Ker-

8) Jahn Arch. Ztg. 1867 S. 44 f. hat die Situation natürlich ganz richtig aufgefaßt, desgleichen Winkler (Bresl. philol. Abhandl. III 61); die seltsamen Deutungen Minervinis und Stephanis auf Aphrodite und Adonis oder Hypermnestra und Lynkeus wird anch Milchböfer nur aus dem Princip des Deutens um jeden Preis erklären kőnnen.

ๆ) Mit Aphrodite auf diesen Darstellungen zu operieren sollte man allmählich doch aufhören; mit welchem Recht darf man diese durch nichts charakterisierte Figur so bezeichnen? Die Frau auf E $\nabla$, die Milchböfer auch als Aphrodite faßt, ist durch das über ihr abgebildete Gefäß deutlich genug gekennzeichnet: sie stammt aus einem größern Gemälde, in dem sie eine Danaide war. Oder enthält E IV zwei Aphroditen?

10) Vgl. S. 388 A. 5. 
beros und Orpheus richtet eine Bitte an Persephone: das sind zwei Handlungen, die in der durch verschiedene Gestalten charakterisierten Unterwelt auf diesen Bildern (E I und E III 1) dargestellt sind; das ganze ist in keinem der beiden Fälle ein bloßes Situationsbild. Die Thatsache, daß wir zwei Heroen im Hades in Thätigkeit sehn, hab ich historisch so zu erklären gesucht, daß einem älteren, in Attika geprägten Unterweltsbild, das die Entführung des Kerberos vorstellte, Orpheus mit seinen Mysten von den unteritalischen Künstlern eingefügt wurde. Der Typus des leierspielenden Orpheus ist natürlich ebenfalls älteren hellenischen Darstellungen entnommen, worauf ich hinzuweisen auf S. 112 meines Aufsatzes nicht unterlassen habe; wir kennen ihn so zwar nicht in der Eurydikescene, wohl aber unter den Thrakern, die er durch seinen Gesang bezaubert.

Ich fasse noch einmal die Argumente für meine Deutung gegen Milchhöfer kurz zusammen. Durch ihre Ausrüstung sind die Jünglinge auf E III 1 und VI 2 deutlich als auf einer Reise befindlich charakterisiert; sie stehn vor den Unterweltsgottheiten, der eine den rechten, der andre beide Arme erhebend. Sie haben also ein Anliegen an Hades und Persephone, worauf auch ihre Bekränzung weist, durch die sie als flehend den Hadesgöttern nahende bezeichnet sind; der Gegenstand einer Bitte an die Hadesgottheiten kann aber nur das Loos des Bittenden nach dem Tode sein. Finden wir auf E III 1 nun auf der andern Seite des Palastes Orpheus, so kann er hier, auf einem unteritalischen Vasengemälde, das einer Blü:hezeit orphischer Religion angehört, nur als der Stifter der Mysterien gedacht sein, der die Bitte seiner Geweihten bei den Unterweltsgöttern unterstützt. Dasselbe gilt für E I, wo er vor einer ganzen Familie von Geweihten steht; durch seine Gegenwart sind die Mysten unzweideutig als orphische bezeichnet. Wollte ein Maler die Scene mit Eurydike darstellen, so malte er die Heroine wie auf E III $2^{11}$ ); ein Eingeweihter fehlt auf diesem Bild, weil Orpheus hier eine andre Aufgabe hat. Das nur aus vier Figuren bestehende Gemälde E $\mathrm{V}$ ist ein Aussehnitt aus einer größeren Darstellung (die Am-

${ }^{11}$ ) Und auf dem Karlsruher Fragment E VI 3, wenn Eurydike dort sicher zur Darstellung der Unterwelt gehörte. 
phora über der weiblichen Figur links weist wie schon hervorgehoben auf eine Danaide), die bestimmt das Objekt von Orpheus Bitte angegeben haben wird, mag es sich nun um einen Geweihten oder um Eurydike gehandelt haben.

Ich möchte bei dieser Gelegenheit noch auf ein bisher nicht genügend erklärtes Bild dic Aufmerksamkeit richten, das bei Raoul Rochette Mon. inéd. pl. 78 abgebildete Gemälde einer unteritalischen Amphora, deren Rïckseite zwei Frauen um eine Grabstele beschäftigt zeigt. Die Vorderseite gliedert sich in zwei Reihen, oben sitzen drei Götter, Athena, Apollon und Artemis; die untere Reihe ist offenbar eine Unterweltsdarstellung, links am Ende steht Persephone neben einem großen Becken wie auf E V, in der Mitte sitzt Pluton auf einem Sessel, ein Scepter in der Rechten. Vor ihm steht in reicher Tracht ein bärtiger mit einem Kranz geschmückter Priester, dessen Haupt bis auf das Antlitz bedeckt wird von einem langen Tuch, das mit Ausnahme der Brust auch seine ganze Gestalt umhüllt. Die erhobene Rechte ruht auf einem mit einer Tänie und Wollbindeu geschmückten Stab mit einer kleinen Cista oben; mit der Linken faßt er eine - gleich den Adoranten - viel kleiner gebildete männliche Gestalt, die ebenfalls ein Himation trägt und mit der Linken einen Zweig erhebt. Nach dem bisher Auseinandergesetzten scheint es mir ganz evident zu sein, daß wir in dem Jüngling einen Eingeweihten zu erkennen haben, der von dem Priester dem Herrscher der Unterwelt zugeführt wird. Ob wir in dem Priester Orpheus sehn dürfen, muß ich einstweilen dahingestellt sein lassen; er mag auch in dieser Gestalt dem Orphiker sofort kenntlich und geläufig gewesen sein. Das Geräth in seiner Rechten kehrt in ähnlicher Gestalt wieder auf der interessanten schwarzfigurigen attischen Vase in Neapel (aus dem Ende des sechsten Jahrhunderts, Heydemann, Katalog der Neapler Vasensamml. 3358), die in den Annali 1865 tav. F abgebildet ist. Stephani hat sie bereits richtig gedeutet, Compte rendu pour 1868 S. 160 . Unter einem Baum sitzen zwei weibliche Gestalten, in denen wir Demeter und Kore zu erkennen haben werden, vor ihnen steht ein Tisch und darunter ein Korb mit Opfergaben. Links von dem Tisch hält ein bärtiger Mann,

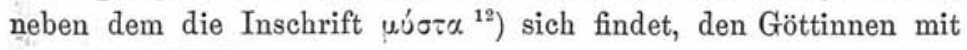

$\left.{ }^{12}\right)$ Die Inschrift ist vollständig; die dorische Form hat nichts 
der Rechten eine Kylix entgegen; in der Linken trägt er auBer einem Schlauch und Zweigen jenes Baums einen langen Stab, auf dem sich ebenfalls eine kleine, unten mit kurzen Bändern geschmückte Cista befindet; ihre Doppelthür ist geöffnet, doch im innern nichts zu erblicken. Wir sehen ein feierliches Opfer vor uns, das den beiden Göttinnen von Eleusis von einem in ihre Mysterien eingeweihten dargebracht wird.

Endlich möchte ich an dieser Stelle eine Beobachtung nicht zurïckhalten, die möglicherweise von einer ganz neuen Seite aus die Erkenntnis der polygnotischen Nekyia fördern kann. Es ist eine auffallende bei der Besprechung dieses Gemäldes nicht berïcksichtigte Thatsache, daß der Unterwelt die Hadesgottheiten Pluton und Persephone ganz fehlen. Wenn die homerische Nekyia sie nicht beschreibt, so findet das seine Erklärung in deren Anlage: an seiner Grube erscheinen dem Odysseus die einzelnen Schatten; von den Frauen ist ausdrücklich bemerkt, daß Persephone sie zu dem Helden sandte (Vers 226). Von dem Maler aber, der die ganze Unterwelt vor unsere Augen stellt, erwartet man, daß er die Herrscher des Schattenreichs uns nicht vorenthalte. Können hier ganz abweichende Vorstellungen zu Grunde gelegen haben? Daß Orpheus in seiner thrakischen Heimath eine ganz andre Rolle spielte als nur die eines Propheten, wird wohl Niemand bezweifeln; Wide ${ }^{13}$ ) schon hat aus seiner Verbindung mit anderen griechischen Gottheiten geschlossen, daß er eine ältere Göttergestalt, ein Hadesgott gewesen sei, der später zur Herrschaft sich durchringenden neuen Culten nicht Stand halten konnte und im Gefolge siegreicher

auffallendes, wenn wir an einen Eingeweibten von Eleusis denken, was bei diesem attischen Gefäß ohnehin anzunehmen ist; in Eleusis hieß die Göttin $\Delta$ xúxтnp, vgl. v. Wilamowitz Aus Kydathen, philol. Unters. I S. 130. A. 50. In diesem Zusammenhang erklärt sich auch

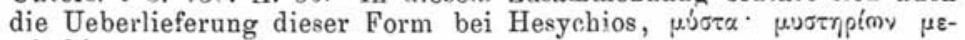
$\tau \alpha \lambda \alpha \beta \omega \dot{\alpha}$.

${ }^{13}$ ) Lakon. Culte Leipz. 1893 S. 140, 174, 244, 295. Vgl. Dieterich Nekyia S. 73. (Als "menschliches Abbild des Zagreus" bezeichnete Orpheus treffend schon 0 . Müller 'Orchomenos' S. 376; ähnlich M. Mayer, 'die Giganten' S. 290 u. A., die Wide bätte citieren können. Soweit sich die griechischen Anschaungen wirklich geschichtlich verfolgen lassen, ist gerade die Mittelstellung des Orpheus als Heros diejGrundlage seiner religiösen Bedeutung. Wide ist in der Creïrung 'alter Götter' vielfach zu weit gegangen, vgl. das litt. Centralbl. 1894, 2, 63. Cr.] 
Gottheiten zum Heros und Mysterienpriester herabsank. Auch die Rolle, die er in den Mysterien spielte, die bis ans Ende der hellenischen Welt seinen Namen trugen, führt auf die Vermuthung, daß er selbst ursprünglich der Unterweltsgott war; auf die Parallele seines Todes mit der Zerreißung des Dionysos durch die Titanen ist bereits von anderer Seite hingewiesen, vgl. Voigt in Roschers mythol. Lex. I Sp. 1421. Der Name seiner Gemahlin Eurydike erinnert an sonstige Bezeichnungen von Hadesgottheiten, wie Eủpúvouos, "Atòns sủpußins (Anthol. Pal. VII $\left.599)^{14}\right)$; auf den von Hermesianax natürlich nicht aus eigner Erfindung ihr beigelegten Namen 'A protír, (vgl. Lobeck Agl. I 373) fällt nun ein ganz andres Licht. Polygnot kam aus Thasos; hat er dort von Thrakien her eine ganz andere Unterweltsvorstellung empfangen, nach der Orpheus als der Herr der Todten galt? Eine Stelle des Pausanias ist bei der Reconstruction des Gemäldes allerseits falsch verstanden worden X

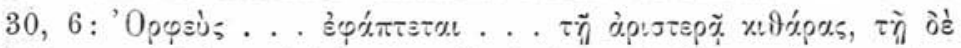

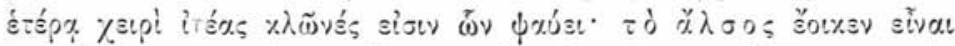

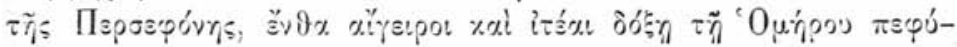
xx.テtv. In der Sprache des Pausanias bedeutet das, daß der Hain der Persephone dargestellt, nicht etwa nur durch einen Baum angedeutet war; über zwei ähnliche Stellen, die darüber keinen Zweifel mehr lassen werden, habe ich in meinem Aufsatz über Statue und Ort in den Jahrbb. f. Philologie Supplementband XIV 277 A. 1 ausführlicher gehandelt; jede Conjectur ${ }^{15}$ ) ist zu vermeiden. Nun erweitert sich das Bild nach rechts und Orpheus rückt mehr in die Mitte; als den inneren Mittelpunkt der Darstellung hat ihn bereits Schreiber ${ }^{16}$ ) in Anspruch genommen. Für die Auffassung der Heroen ist damit freilich nicht viel gewonnen; denn in ihnen die seligen an des Silenos Flötenspiel sich erfreuenden Anakten erkennen zu wollen, wie Schreiber es thut, dem widerspricht der ganze stille Charakter jener Welt, den Schöne ${ }^{17}$ ) meiner Empfindung nach durchaus

14) Vgl. Hesiod Theog. 239 Eupußi हैyouøa. Auch der auf einer Weihinschrift im Piraeus erwähnte Heros

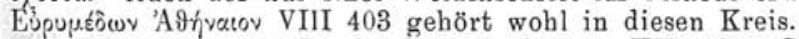

$\left.{ }^{15}\right)$ Vgl. Schöne, Jahrb. d. archäol. Inst. VIII 1893 S. 211 A. 49.

${ }^{16)}$ Festschrift für Overbeck 1893, S. $204 \mathrm{ff}$.

17) A. a. O. S. $202 / 3$. 
richtig gezeichnet hat. Aber einen Schritt weiter in der Erkenntnis der mythischen Grundlagen unseres Bildes würde meine Auffassung, wenn sie die richtige ist, doch führen und ich glaubte sie daher zu ernstlicher Erwägung vorlegen zu dürfen. Sie würde endgültig davor bewahren, zur Erklärung des delphischen Gemäldes die Mysterien von Eleusis heranzuziehn, einen Versuch den sich ja selbst die unteritalischen Vasenbilder haben gefallen lassen müssen; ich kann es nicht unterlassen, zum Schluß noch kurz die Kritik, die Milchhöfer in dieser Beziehung an Dieterich geübt hat, zu berühren. Pausanias allerdings hat in den Uneingeweihten des polygnotischen Bildes eleusinische zu

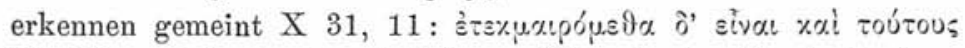

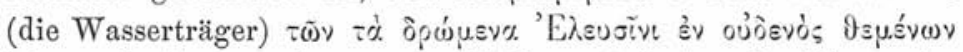

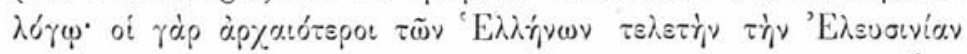

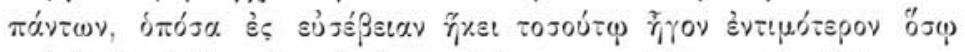
xai daß er sich damit nur eine Conjectur erlaubt; Dieterich hat sie als eine falsche zurückgewiesen, weil die Strafe des Wassertragens als orphische Lehre bereits im vierten Jahrhundert überliefert wird. Milchhöfer hält des Pausanias Vermuthung für richtig, „das beweisen auf der andern Seite (des Gemäldes) die Geweibten der Demeter im Nachen des Charon" (S. 392). Als ob es nicht genug andre Demetermysterien gegeben hätte, als grade die eleusinischen; von Kleoboia sagt Pausanias zum Ueberfluß XXVIII 3 ausdrücklich, daß sie die Demeterorgien nach Thasos aus Paros gebracht habe, also nicht aus Eleusis. Daß Dieterich ja selbst für die ältere Zeit orphischen Einfluß auf Eleusis nicht annähme, genügt doch nicht zu seiner Widerlegung (S. 392, 8); denn Niemand wird dem Pausanias zutrauen, $\mathrm{daB}$ er eine genaue Kenntnis vom Zustand der eleusinischen Mysterien im fünften Jahrhundert gehabt und diese seinem זะxuraípsof wicklung, sondern urtheilte nach den Vorstellungen, die zu seiner Zeit in Eleusis herrschten, und deren Beeinflussung durch orphische Lehren hat Dieterich Nekyia S. 75, 2 hervorgehoben. Eine Verwechslung von eleusinischem und orphischem liegt also bei Plato nicht vor: es war ein vergeblicher Aufwand von Scharfsinn, der in dem Sieb das unterscheidende, specifisch orphische erkennen lieB.

Marburg.

Ennst Kuhnert. 